\title{
The brain decade in debate: VI. Sensory and motor maps: dynamics and plasticity
}

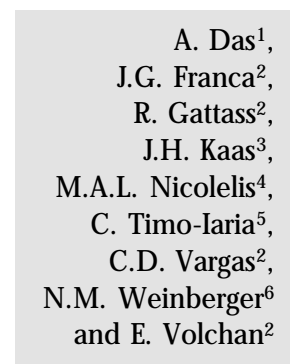

\author{
${ }^{1}$ Department of Neurobiology, Rockefeller University, New York, NY, USA \\ IInstituto de Biofísica Carlos Chagas Filho, \\ Universidade Federal do Rio de Janeiro, Rio de Janeiro, RJ, Brasil \\ ${ }^{3}$ Department of Psychology, Vanderbilt U niversity, Nashville, TN, USA \\ ${ }^{4}$ Department of N eurobiology, D uke U niversity, Durham, NC, USA \\ ${ }^{5}$ Departamento de Neurologia, Faculdade de Medicina, \\ Universidade de São Paulo, São Paulo, SP, Brasil \\ ${ }^{6}$ Center of Neurobiology, Learning and Memory, University of California, \\ Irvine, CA, USA
}

\section{Correspondence \\ E. Volchan \\ Laboratório de Neurobiologia II \\ Instituto de Biofísica Carlos \\ Chagas Filho, UFRJ \\ CCS, Bloco G, Sala G2, 35 \\ 21949-900 Rio de Janeiro, RJ \\ Brasil \\ E-mail: evolchan@ biof.ufrj.br \\ The names of the authors are presented in alphabetical order, except for the last one that corresponds to the symposium organizer.}

Publication supported by FAPESP

Received July 11, 2001

Accepted August 20, 2001

\section{Abstract}

This article is an edited transcription of a virtual symposium promoted by the Brazilian Society of Neuroscience and Behavior (SBNeC). Although the dynamics of sensory and motor representations have been one of the most studied features of the central nervous system, the actual mechanisms of brain plasticity that underlie the dynamic nature of sensory and motor maps are not entirely unraveled. Our discussion began with the notion that the processing of sensory information depends on many different cortical areas. Some of them are arranged topographically and others have non-topographic (analytical) properties. Besides a sensory component, every cortical area has an efferent output that can be mapped and can influence motor behavior. Although new behaviors might be related to modifications of the sensory or motor representations in a given cortical area, they can also be the result of the acquired ability to make new associations between specific sensory cues and certain movements, a type of learning known as conditioning motor learning. Many types of learning are directly related to the emotional or cognitive context in which a new behavior is acquired. This has been demonstrated by paradigms in which the receptive field properties of cortical neurons are modified when an animal is engaged in a given discrimination task or when a triggering feature is paired with an aversive stimulus. The role of the cholinergic input from the nucleus basalis to the neocortex was also highlighted as one important component of the circuits responsible for the context-dependent changes that can be induced in cortical maps.
Key words

- Visual

- Somatosensory

- Auditory

- Motor

- Cortex

- Topography

- Learning

- Synesthesia 


\section{Introduction}

Since the early electrophysiological studies describing sensory "maps" on primary cortical areas (vision (1), hearing (2), touch (3)) the search for brain maps has occupied center stage in system neurophysiology. Areas can be distinguished because their unique functional roles require structural specializations, unique patterns of input and output and neurons with specific ways of responding to stimulus events (4). In primates, although investigators still disagree about the precise number of sensory and motor areas, since more areas are being discovered and some proposed fields are in question, there is good agreement that the visual cortex of monkeys includes more than 30 visual areas, the somatosensory cortex has at least 10 , the auditory cortex has 12 or more, and the frontal cortex contains approximately 8 to 10 motor areas (see Ref. 5). There is now overwhelming evidence that maps are not fixed or immutable in the adult brain. Studies of adult brain plasticity are revealing how the brain operates in functional compensations, in improving the performance of sensory motor skills and perceptual abilities, in allowing recalibrations of sensory systems after receptor surface damage, and in mediating recovery after central nervous system damage. As plastic changes in sensory and motor maps seem to be a critical component of learning new skills and providing the ability to make novel sensory discriminations, it has been proposed that plastic processes form a routine part of cortical computation (6).

In a recent paper, Ghazanfar and Nicolelis (7) called attention to the fact that under natural conditions animals must process spatiotemporally complex signals in order to guide adaptive behavior. The authors reflect that map plasticity not only engages "local" circuits but often a more "systemic" activation of broadly related neural structures. As an example, the work of Weinberger (8) showed at the primary auditory cortex level an enlarged representation of a tone that was aversively conditioned. Such plasticity is thought to involve the medial geniculate of the thalamus and the cholinergic system of the basal forebrain (9).

This "system" view is being more and more accepted by different research groups since cumulative evidence points to brain work in an integrated fashion. Cross-modal activity is being revealed even in primary sensory and motor areas, and emotion, attention, motivation and arousal seem to be crucial for the normal processing of both primary and non-primary regions.

In order to discuss the state of the art in the dynamic nature of sensory and motor brain maps, the Brazilian Society of Neuroscience and Behavior ( $\mathrm{SBNeC})$ promoted this virtual debate as part of the series in which the main achievements of the so-called "Brain Decade" are revisited. Some active researchers in the field were invited to the present symposium which was held on December 8, 2000 at a chat site provided by the Conselho Nacional de Desenvolvimento Científico e Tecnológico (CNPq). The present article is a transcription of this symposium.

Eliane Volchan: We plan to discuss the following topics: 1) What is a map in the brain? What are they for? 2) How plastic can a map be? 3) Is synesthesia related to brain maps? 4) Are emotions involved in sensory and motor map dynamics? Ricardo might start talking about topography in visual maps.

Ricardo Gattass: We propose a framework for understanding visual perception based on a topographically organized, functionally distributed network. In this proposal the extraction of shape boundaries starts in retinal ganglion cells with concentric receptive fields. This information, relayed through the lateral geniculate nucleus, creates a neural representation of negative and positive boundaries in a set of topographically con- 
nected and organized visual areas. After boundary extraction, several processes involving contrast, brightness, texture and motion extraction take place in subsequent topographically organized visual areas in different cortical modules. Following these processing steps, filling-in processes at different levels within each area and in separate channels propagate locally to transform boundary representations to surface representations. These partial representations of the image propagate back and forth in the network, yielding a neural representation of the original image. It has been proposed that cortical topography minimizes the total volume of axons and dendrites (10). The local computations supported by topographic maps might then favor the integration of information that preserves spatial structure. In such cases, filling-in would be accompanied by what seems like spreading interactions and spatially organized representations. Moreover, effective use of the cortical circuitry would be made. In summary, in the task of organizing the scene into context-dependent meaningful representations, the visual system relies both on local estimates of image attributes (such as contrast) and on integration mechanisms that operate in topographically organized areas (which are in some cases associated with perceptual filling-in). Integration need not be confined to a single cortical area. Instead, given the highly interconnected array of visual areas, it is likely that they cooperate in the task of integration, forming dynamic and context-dependent coalitions of areas. Thus, the multiple organizations of the stimulus that are possible are organized into a single coherent one that is used to effectively guide behavior.

Eliane Volchan: Ricardo, how do you compare topographic and non-topographic representations in a single sensory system, say vision?

Ricardo Gattass: It has been proposed that different visual attributes are segregated and processed in different extra-striate vi- sual areas $(11,12)$, while the notion of extrastriate areas with analytical properties and inferotemporal areas with synthetic properties was proposed by Gross et al. (13). Charles Gross's model separates topographically organized extra-striate areas (analytic) from non-topographic ones (synthetic). We enhanced Charlie's model by introducing the concept of a topographic distributed network in a number of extra-striate areas (14), containing organized maps of the visual field with different functional modules for visual processing (e.g., blobs in $\mathrm{V} 1$ and cytochrome oxidase-rich stripes in V2) should be contrasted to other types of organization that involve non-topographically organized structures. Examples of such non-topographic analytical areas are those of the intraparietal sulcus (LIPv and LIPd) where remapping of the visual information related to the movement of the eyes takes place (15) or those of the parieto-occipital cleft (areas PO and POd), where the visual maps are organized along the isopolar dimension and not along the isocentric dimension (16).

Eliane Volchan: Miguel, would you like to say something about motor maps?

Miguel Nicolelis: Eliane, I think I would start by saying that in the motor cortex the concept of a map is not as straightforward as in primary sensory cortices.

Eliane Volchan: What are the main differences?

Miguel Nicolelis: Well, although one can identify topographic relationships in both motor and sensory maps, in motor cortex there is a hot debate regarding what is represented there.

João Franca: Miguel, are you talking about the problem of what is represented in motor cortices (i.e., movement versus muscle)?

Miguel Nicolelis: Yes, João, that is one of the issues. The other issue is that you can activate the same part of the body by stimulating different locations of a motor cortical area. Actually, Dr. Kaas has studied both 
sensory and motor maps and could talk a lot about such comparisons.

Jon Kaas: Maps reflect an orderly arrangement of inputs, and the inputs can represent anything - receptor surfaces, afferent inputs, or central computations. Maps reflect a global organization, but within a map there can be modular organization as in the motor maps (17).

João Franca: Do you consider the paradigm of mapping sensory versus motor areas really comparable? It seems to me that the variables of a sensory stimulus are better controlled than those of an electrical stimulation in the cortical tissue.

Jon Kaas: Motor maps are output maps, but the sensory maps are input maps. So at that level they are not strictly comparable. But the motor maps have a sensory component that can be mapped, and sensory maps may also contain motor maps.

Eliane Volchan: Jon, do we only consider as "map" the sensory responses in motor cortex (is this what you meant)?

Jon Kaas: Eliane, the organization of inputs to layer 4 determines the organization of maps in any area. Both output and input maps reflect the organization of connections. The sensory map largely overlaps the motor maps, and the sensory areas also contain motor maps. So both input and output maps are 'maps'.

Ricardo Gattass: Dr. Kaas, the visual maps of PO may reflect another type of organization other than organization of connections. This principle may apply to early areas.

Eliane Volchan: Can you explain it, Ricardo?

Ricardo Gattass: For this discussion, I will use the data of Neuenschwander et al. (16) on the visuotopic organization of visual area PO to trace a parallel between map and function. The visual field representation in areas PO and POd is complex. These areas have an almost complete representation of the contralateral visual hemifield, which is more regularly organized in the isopolar dimension. At present, we have no direct evidence regarding the functional significance of the observed order in the isopolar domain versus disorder in the isoeccentric lines present in PO and POd. One may speculate, however, that centrifugal and centripetal organizations of directionality, such as those observed in area PG $(18,19)$, demand interactions between neurons that analyze regions of space sharing a similar polar angle but with different eccentricities. Also, during forward egocentric motion, the angle of gaze is the center of the expanding flow field that shows some constancy in the isopolar domain while presenting large changes in the isoeccentric domain. It may be that the intermixing of eccentricities of receptive fields in adjacent columns in PO and POd allows these interactions to occur by local circuits. Thus, the visual maps of PO and POd may be the consequence of different computational strategies used by these areas. The homogeneous representation of the visual field periphery in PO and POd may provide the essential inputs mediating flowfield perception during animal locomotion. In addition, stimulation of the visual field periphery was shown to dominate the perception of self-movement, even when antagonistic moving images are presented at center of gaze (20). Therefore, one could suggest that these areas with little central representation might be involved in visuomotor integration and perception of global spatial relationships. Interestingly, PO, POd and PG were shown to project to the pontine nuclei, which relay information to the part of the cerebellum involved in motor planning (21).

Miguel Nicolelis: Ditto. I would just add that maps reflect one of the many potential levels of organization of a particular cortical area. Thus, within this global structure one can identify other principles of organization. Wow! That was quite a statement Ricardo. Brazilian style!! 
Cesar Timo-Iaria: The last comment by Dr. Gattass is very interesting and I would like to add a few words to it. I have a theory to explain not only the incredibly complex network of pathways that go to or leave the cerebellum but also the involvement of this organ in all kinds of neural functions, from modulating motoneurons (a subject I studied in the sixties) to influencing learning, late components of event-related evoked potentials in neocortical areas and conscious functions, and to intervening in sleep physiology (as we are finding currently). The cerebellar cortex is well known to receive information from all sensory channels, including the carotid sinus baroreceptors. It receives a huge projection from all cortical sensory and motor fields, as well as from the basal ganglia, and sends information to all these areas. We might reason that when a given system programs a behavior it sends the program to the cerebellum, which in turn receives from the effector organs information about how the program is being performed; the program and its performance are dynamically compared on-line and the discrepancies are corrected by the cerebellar efferent pathways to the effector neurons, including the vegetative components of any behavior. In 1938, Moruzzi (22) demonstrated that electrical stimulation of the anterior lobe of the cerebellar cortex inhibits the baroreceptor reflex, which is known to be involved in the blood pressure increase as a component of most behaviors. The classic index of fingernose poor performance, the characteristic "cerebellar" speech, the awkward and unstable walking, as well as astasia and abasia that do unveil a cerebellar lesion in humans are fully accounted for by the theory. The finger-nose test is performed and, like speech, walking and fundamental posture, can be produced but quite imperfectly when the cerebellum is lesioned; the core of the behavior is there but not the corrections.

Eliane Volchan: How far does the motor overlap go in sensory systems? Up to pri- mary areas?

Jon Kaas: Eliane, all areas have subcortical outputs, but you cannot always elicit movement easily. For example, all visual areas project to the superior colliculus to influence eye movements, but that influence may not be apparent during motor mapping experiments.

Claudia Vargas: Miguel, about motor maps: Could the temporal dynamics of plasticity in motor maps be much more related to what movements are actually being executed? Or can a neuron in the motor cortex have a multiple representation of movement?

Miguel Nicolelis: Claudia, there is nice evidence suggesting that single neurons in the motor cortex are definitely involved in the control of multiple muscles (23). I believe that these neurons may participate in the computation of many motor parameters simultaneously. Motor cortical plasticity also seems to be driven by changes in the animal's experience, so in that sense changes in motor experience are an important driving force for plastic reorganization in the motor cortex. In essence I was trying to say that I agree with you. The dynamics one observes in motor maps seems to reflect the type of movements made by the animal, and this inherent dynamics provides the substrate, the driving force for plastic reorganization.

João Franca: It is already well established that brain maps, even in primary sensory areas, change as a result of lesions or learning (for a review, see Ref. 6). But what are the actual mechanisms of brain plasticity? Are they the same for all cortical areas? Back to the case of motor maps: If new movements imply new representations in motor areas, are they instantaneously generated by the cortical circuitry? How timedependent are the changes involved? What are the mechanisms involved in these changes?

Miguel Nicolelis: João, we know that motor plasticity is not necessarily limited to the production of new movements. It can 
also be reflected in the acquired ability of arbitrarily associating sensory cues with different movements. In this scenario, the movements are the same, but the combination of sensory cues that triggers them has changed. In this type of learning, also known as conditional motor learning, the output does not change, only the arbitrary sensory-motor mapping does (for a review, see Ref. 24).

Claudia Vargas: Jon, but what about the maps "of internal use"? Those that are used to build the re-representations? For instance, those in non-topographic analytical areas cited by Ricardo?

Jon Kaas: Claudia, can you expand on your question please?

Claudia Vargas: In line with Damasio's work (25), what is the relationship between the sensory maps and the subjective body representation? How does it relate to the online corporal awareness?

Eliane Volchan: A recent study by Damasio's group (26) has shown that lesions to the somatosensory cortex impair the visual recognition of a facial emotional expression. The authors discuss the idea that this recognition may not necessarily need the production of facial mimicry by the observer, in line with Damasio's idea of "as-if" loops (27).

Cesar Timo-Iaria: When we pay attention to anything, either in the outside world or in the inside mental world, we always produce a motor output, at least the ocular muscles do. We always at least immobilize the eyes when we are paying attention, including when we are calculating or building up a phrase or a thought. I think this motor component is enough to characterize paying attention or thinking as specific behaviors.

Claudia Vargas: Jon, in some of the above mentioned dorsal stream areas, for instance, the map of the surface representation seems to be space-transformed so as to allow appropriate effector movements. In those areas, space is coded in body-centered frames of reference, anchored now to the motor output (for reviews, see 28,29).

Jon Kaas: Claudia, after reorganization the sensory map may often differ from the perceptual map (30). Probably feedback is used to align sensory and perceptual maps.

Eliane Volchan: Jon, what do you mean by perceptual map?

Jon Kaas: Eliane, all I mean is that within a map if you stimulate, you get perception of the receptor surface that normally corresponds to the sensory map. So you could ask a person what she feels and get a perceptual map, or you could generate a sensory map by stimulating the peripheral receptors. Perception involves whole systems and many maps (17). When you electrically stimulate one map you are provided output to other maps.

Claudia Vargas: Jon, do you mean that subjective corporal awareness would be more related to perceptual maps? Is there a second level of representation there, which includes the primary maps? On which grounds? A map of maps?

Ricardo Gattass: Jon, how do you conciliate perceptual maps with the absence of topography in synthetic areas such as the inferotemporal cortex. Where are the "internal models" located?

Miguel Nicolelis: Ricardo, my only point in response to your question is, why does one need to equate topography with perception? The olfactory cortex is not topographically organized and yet rodents and primates can smell bad food very fast!!

Aniruddha Das: Claudia, I suppose also that we should think of these perceptual maps really as a model for actions that the animal is planning, i.e., not as "passive" analysis of input per se (many people take it as an article of faith that this is the most important aim of the sensory system, and pure sensory perception is only a partial answer). So, in that sense, I don't know what you might be thinking of as a "map of maps", but one should consider that the "model" might be at this level of mapping onto the desired action. 
Claudia Vargas: Yes, Aniruddha. So in this sense, maybe we could think about a similarity in the plastic modulation of sensory and motor systems, based on the planning of the "desired action" and then we go to the last point Eliane has mentioned, that is, to what extent is there an emotional modulation over the sensory and motor systems.

Ricardo Gattass: Aniruddha, before we finish please comment: Our proposal for cortical computation emphasizes the network of connections that engage topographically organized areas. Feed-forward connections topographically link areas, while a much more disperse feedback arrangement is also present at all levels, including the striate cortex (V1). According to the present proposal, distinct forms of perceptual completion engage different portions of the widely distributed processing network. For example, line completion of the type triggered by the large bar stimuli of the Fiorani Junior et al. (31) study, most likely receive a central contribution from striate cortex circuits as revealed by the cell responses obtained in this area. At the same time, boundary completion associated with illusory contour stimuli probably involves V2 circuits as revealed by the studies of von der Heydt and Peterhans (32). These two forms of boundary completion should be contrasted with feature completion, such as brightness and texture fillingin. The results from the work of De Weerd et al. (33) strongly suggest that V2 and V3 (but not V1) mediate texture filling-in. At the same time, brightness filling-in seems, at least in part, to involve the striate cortex (34). However, the view we advance here is not the "one effect-one area", but rather that a distributed network of topographically organized areas is involved. So, for example, $\mathrm{V} 2$ and V3 circuits are important for texture filling-in. At the same time, while V1 was implicated in brightness coding by Rossi et al. (34), several other areas are likely to be involved too, such as V4 whose cells display sophisticated color-coding properties. Fi- nally, although illusory contours trigger responses in V2, recent evidence suggests that other areas may be involved also (such as V4).

Aniruddha Das: Hi, Ricardo - I agree with you that one must consider this combined feed-forward and feedback process, and not "one area-one effect". Many of these higher order perceptions might be "triggered" in the extra-striate cortex, in V2 for illusory contours, etc., but presumably with a feedback to V1 and you could possibly see consequences of that in increased firing or temporal correlation. For example, even with such higher order effects as attentional modulation, you do get distinct effects in V1 at the single cell level (35) (that interacts with contour integration in V1) as well as in the general increase in "background/spontaneous" activity that shows up in functional magnetic resonance imaging studies of $\mathrm{V} 1$ activity with difficult tasks $(36,37)$.

Eliane Volchan: We have been dealing with the first and second questions. Maybe we could go on to synesthesia. I add the following question: Ramachandran and Rogers-Ramachandran (38) have shown that amputees can have a "vivid" somatic perception of a phantom arm by visualizing it through a mirror. Are proprioception and vision synesthetic senses in humans? Do other animals have other combinations of senses, like olfaction and vision or hearing and vision?

Miguel Nicolelis: Eliane, I think that there is no doubt that the network of cortical areas and thalamic nuclei that form the somatosensory system contains an internal model of the body. This "internal model" seems to be crucial to define the limits of the body and relationships between our bodies and extrapersonal space. I think the phantom-limb phenomenon illustrates what may happen when this "internal model" is confronted with the lack of input from a part of the body that should be there. This may create a mismatch that creates the illusion that the miss- 
ing limb is still there!

Jon Kaas: Eliane, the perception of the phantom in cases with congenital missing limb can perhaps answer this question $(39,40)$, but the claim that phantoms exist in such cases is still very controversial.

Miguel Nicolelis: Eliane, it is really difficult to judge Ramachandran's experiments. These are pretty subjective descriptions and I am not sure I would build a whole theory based on them. Nonetheless, as you said, this would involve a second sensory modality, vision, reinforcing a somatosensory percept. In this case, I am not sure what the subject is actually feeling, but this description would suggest that the sense of vision could contribute to the definition of an internal body image.

Eliane Volchan: Miguel, but when the person sees the limb in a mirror the phantom becomes much more real including its movements and there is no mismatch but instead a match that can be the same as in normal people (see Ref. 41). That is why I talked about synesthesia. The work on the interaction of visual and kinesthetic perception of normal limbs also highlights the idea of synesthesia (42). It is also in line with recent data obtained with normal subjects indicating the lack of tactile discrimination when the visual cortex is inhibited (43) and activation of the visual cortex during blind-folded tactile discrimination (44).

Miguel Nicolelis: Eliane, it would be really interesting to find out what happens after amputees have interacted with the mirrors for several hours. I would predict that their phantoms go away! What do you think?

João Franca: Miguel, this is actually what happens. After a few weeks of training, some subjects report a "telescoping effect". The phantom limb diminishes, but the more represented parts (i.e., the fingers in the case of an amputed arm) remain attached to the stump (45).

Eliane Volchan: We could address the last question now on the role of emotions in sensory and motor map dynamics. Weinberger (8) showed that the tonotopic map in A1 changes by pairing a tone with an aversive stimulus, i.e., the cortical representation of that tone expands. Is this modulation present in other primary sensory cortices? Is it similar for all mammals?

Eliane Volchan: Aniruddha, do you think the representation of one coordinate in V1 could be magnified if this region is paired with an aversive stimulus?

Aniruddha Das: Eliane, Yves Fregnac and colleagues $(46,47)$ have shown a whole range of similar effects in V1 - where you get a change in the orientation tuning by pairing a particular orientation with stimulation (they used extracellular electrical stimulation, for example), again, you get a shift in the peak orientation tuning but not a bodily shift in orientation tuning, similar to Norman Weinberger's results.

João Franca: Aniruddha, keep in mind the paradigm of enlarging the S1 representation of a skin patch after training a monkey in a tactile frequency discrimination task (48). Do you think that the receptive field properties of V1 cells (such as orientation tuning) could be similarly changed by training?

Aniruddha Das: João, it's conceivable and one point that has come out, for example in plasticity in V1, is that the effects seen on individual neurons depend strongly on the precise task used and you only see them manifested when the animal is engaged in the same task. So, for example, Roy Crist and colleagues $(49,50)$ showed that you could train animals (including humans) to get better at a 3-line bisection task but not a hyperacuity task, even though the two tasks use the same vertical lines in the same region of the cortex. Later, the same subject can be independently trained to get better in the hyperacuity task. Further, by working with animals, Roy showed that a) after training with a 3line bisection task, the cells in the trained hemisphere showed a change in their modulation, i.e., now showed facilitation rather 
than suppression with parallel flanking lines. This was true only in the trained region and not in an untrained region; b) very interestingly, these cells showed this effect only when the animal was engaged in the same 3line bisection discrimination task. The same stimulus pattern, presented during passive viewing, showed no such change in the modulation. So, the changes are both task-specific, and somehow controlled so as to come into effect only within a particular task context.

Eliane Volchan: Dr. Weinberger, we were discussing your results on the magnification of the tone paired with an aversive stimulus in the primary auditory cortex and wondering if the same could happen with other systems.

Norman Weinberger: We study the primary auditory cortex (A1) not the visual cortex (V1). So far, researchers have found similar effects in the somatosensory cortex. I have discussed the visual cortex with some visual physiologists, who are split. Some seem to think V1 is not plastic but I think this reflects unwarranted assumptions. Certainly, studies of visual learning implicate V1 (51). The learning experiments are simple to do in animals and should be done.

Eliane Volchan: Norman, Aniruddha mentioned experiments by Roy Crist and Charles Gilbert in the primate visual system but none was done with an "emotional" stimulus.

Norman Weinberger: I cannot access previous messages for some reason, but I will add a comment on the issue of what parts of the cortex are plastic in learning. There is good reason to believe that the nucleus basalis cholinergic projections to the cortex enable retuning and other plasticities under circumstances of learning (8). There is every reason to believe that all areas of the cortex can participate. We selected the auditory cortex because of tactical advantages not because it was thought to be uniquely plastic.
João Franca: This is interesting. The nucleus basalis does not project uniformly to all cortical areas. Actually, occipital (visual) areas receive less cholinergic projections than the parietal and frontal areas (52). Does it mean that plasticity shall be different in these different sensory areas (i.e., visual versus somatosensory or auditory areas)?

Norman Weinberger: It is known that V1 is subject to modulation by acetylcholine. I think we need to be careful in associating the amount of anatomical projections with the amount of physiological influence. McGaugh and Sarter (53) have shown that saporin in visual cortex produces specific degeneration in the nucleus basalis and specific deficits in what he refers to as visual attention.

João Franca: Norman, the differential distribution of cholinergic projections from the nucleus basalis is followed by a heterogeneous distribution of nitric oxide (NO)producing neurons $(54,55)$. In addition, we showed that there are fewer NO neurons in visual areas compared to somatosensory areas (56). Since NO is crucial for some forms of synaptic plasticity (57-59), I wonder if such anatomical/histochemical heterogeneities could be reflected in the physiological dynamics of sensory maps.

Norman Weinberger: I definitely think that could be the case... I hope you can do some of the sensory map or preferably receptive field studies within the context of NO.

Norman Weinberger: There must certainly be limitations and constraints on plasticity. My guess is that plasticity is much greater than thought but we do not yet have an overall theory that would allow precise predictions about which stimulus parameters would be affected in which tasks. The issue of selective engagement of previously developed changes is critical, as pointed out in this discussion. Behavioral studies have long shown that many aspects of learning and memory are context dependent. From the 
brain's point of view, there is probably no such thing as a stimulus, but simply the totality of afferent sensory influx. Thus, training to a given stimulus may have contextual bounds. A simple example is that our animals do not undergo extinction during determination of receptive fields, although they can extinguish if the same conditioned stimulus tone is presented within the same training context. The context of receptive field determination is completely different. A paper by Diamond and Weinberger (60) addresses this issue.

Eliane Volchan: Norman, that is really interesting since I have been wondering how to test the receptive fields without inducing extinction. Was the animal awake during the recordings?

Norman Weinberger: Receptive field plasticity can be read-out under Nembutal or ketamine if the conditioning first took place in the waking state. All of this is well described in our papers (60-65).

Eliane Volchan: Norman, do you think the direct amygdalocortical projections play a role in the conditioning paradigm?

Norman Weinberger: With respect to the amygdala, it is actually interesting that, e.g., fear conditioning does not require the basolateral amygdala. There is the widespread but not substantiated belief that fear memories are stored in the basolateral amygdala, a position strongly taken by Joe LeDoux (66). In any event, there is not much doubt that the amygdala plays a very important role in the modulation of the strengths of memories, as well attested to by the work of Jim McGaugh and others $(53,67)$. However, such influences require a cholinergic link, still under investigation.

Eliane Volchan: Norman, back to fear conditioning. The problem in the visual sys- tem of non-primates is that we cannot repeat the experiments you did (previous conditioning) because the animal cannot be trained to fixate. I was wondering if a loud sound paired with a visual stimulus would work in an anesthetized and paralyzed preparation, given that changes in heartbeats could be tracked concurrently.

Norman Weinberger: Eliane, I think the main problem is anesthesia. You might be able to induce plasticity in anesthesia by stimulating the nucleus basalis. You might check the work of Dykes (68) in the somatosensory cortex on this. Then, you could control the visual stimuli. It is difficult to establish learning/plasticity in anesthetized subjects and of course there is no behavioral read out. But we did induce fear conditioning in rats with Paul Gold $(69,70)$, when they were under deep barbiturate anesthesia, by giving a small dose of peripheral epinephrine. Days after recovery, subjects exhibited fear.

Norman Weinberger: I am sorry I could join only late. However, I would be most happy to correspond with everyone individually, at any time.

João Franca: I would like to congratulate the $\mathrm{SBNeC}$ for this innovative and lowcost initiative of promoting these virtual discussions.

\section{Acknowledgments}

The authors wish to thank Dr. Claudio da Cunha for great help with the organization of the chat, CNPq-Prossiga for providing the chat web room, and the Brazilian Journal of Medical and Biological Research for the paper and electronic publishing (www. scielo.br/BJMBR) of this text. 


\section{References}

1. Talbot SA \& Marshall WD (1941). Physiological studies on neural mechanisms of visual localization and discrimination. American J ournal of Ophthalmology, 24: 1255-1264.

2. Woolsey CN \& Waltz EM (1942). Topical projection of nerve fibers from local regions of the cochlea to the cerebral cortex of the cat. Bulletin of the J ohns Hopkins Hospital, 71: 315-344.

3. Woolsey $\mathrm{CN}$, Marshal $\mathrm{WH} \&$ Bard $\mathrm{P}$ (1942). Representation of cutaneous tactile sensibility in the cerebral cortex of the monkey as indicated by evoked potentials. Bulletin of the J ohns Hopkins Hospital, 70: 399-441.

4. Kaas J H (1989). Why does the brain have so many visual areas? J ournal of Cognitive Neuroscience, 1: 121-135.

5. Kaas J H (1999). The transformation of association cortex into sensory cortex. Brain Research Bulletin, 50: 425.

6. Das A (1997). Plasticity in adult sensory cortex: a review. Network-Computation in Neural Systems, 8: R33-R76.

7. Ghazanfar AA \& Nicolelis MAL (2001). The structure and function of dynamic cortical and thalamic receptive fields. Cerebral Cortex, 11: 183-193.

8. Weinberger NM (1995). Retuning the brain by fear conditioning. In: Gazzaniga MS (Editor), The Cognitive Neurosciences. MIT Press, Cambridge, MA, 10711090.

9. Bjordhal TS, Dimyan MA \& Weinberger NM (1998). Induction of long-term receptive field plasticity in the auditory cortex of the waking guinea pig by stimulation of the nucleus basalis. Behavioral Neuroscience, 105: 416-430.

10. Cowey A (1979). Cortical maps and visual perception: the Grindley Memorial Lecture. Quarterly J ournal of Experimental Psychology, 31: 1-17.

11. Zeki S (1978). Functional specialization in the visual cortex of the rhesus monkey. Nature, 274: 423-428.

12. Felleman DJ \& Van Essen DC (1991). Distributed hierarchical processing in the primate cerebral cortex. Cerebral Cortex, 1: 1-47.

13. Gross CG, Rocha-Miranda CE \& Bender DB (1972). Visual properties of neurons in inferotemporal cortex of the macaque. J oumal of Neurophysiology, 35: 96-111.

14. Gattass R, Rosa MG, Sousa AP, Pinon MC, Fiorani J unior $M \&$ Neuenschwander S (1990). Cortical streams of visual information processing in primates. Brazilian
J ournal of Medical and Biological Research, 23: 375-393.

15. Duhamel JR, Colby $\mathrm{CL} \&$ Goldberg $\mathrm{ME}$ (1992). The updating of the representation of visual space in parietal cortex by intended eye movements. Science, 255 : 90-92.

16. Neuenschwander $S$, Gattass R, Sousa AP $\&$ Pinon MC (1994). Identification and visuotopic organization of areas $\mathrm{PO}$ and POd in Cebus monkey. J ournal of Comparative Neurology, 340: 65-86.

17. Kaas JH (1997). Topographic maps are fundamental to sensory processing. Brain Research Bulletin, 44: 107-112.

18. Motter BC \& Mountcastle VB (1981). The functional properties of the light-sensitive neurons of the posterior parietal cortex studied in waking monkeys: foveal sparing and opponent vector organization. J ournal of Neuroscience, 1: 3-26.

19. Steinmetz MA, Motter BC, Duffy CJ \& Mountcastle VB (1987). Functional properties of parietal visual neurons: radial organization of directionalities within the visual field. J oumal of Neuroscience, 7: 177191.

20. Tichgans J \& Brandt TH (1974). The psychophysics of visually induced perception of self-motion and tilt. In: Schmidt FO \& Worden FG (Editors), The Neurosciences - Third Study Program. MIT Press, Cambridge, MA, 123-129.

21. Brodal P (1978). The corticopontine projection in the rhesus monkey. Origin and principles of organization. Brain, 101: 251283.

22. Moruzzi G (1938). Azione del paleocerebellum sui riflessi vasomotori. Archivio di Fisiologia, 38: 36-78.

23. Schieber MH \& Hibbard LS (2001). How somatotopic is the motor cortex hand area? Science, 261: 489-492.

24. Laubach $M$, Weissberg J \& Nicolelis MAL (2000). Cortical ensemble activity increasingly predicts behavior outcomes during learning of a motor task. Nature, 405: 567571.

25. Damasio A (1999). The Feeling of What Happens: Body and Emotion in the Making of Consciousness. Harcourt Brace \& Co., New York.

26. Adolphs R, Damasio H, Tranel D, Cooper $\mathrm{G} \&$ Damasio AR (2000). A role for somatosensory cortices in the visual recognition of emotion as revealed by three-dimensional lesion mapping. J ournal of Neuroscience, 20: 2683-2690.

27. Damasio A (1994). Descarte's Error. Gros-
set/Putman, New York.

28. Graziano MSA \& Gross CG (1995). The representation of extrapersonal space: A possible role for bimodal, visual-tactile neurons. In: Gazzaniga MS (Editor), The Cognitive Neurosciences. MIT Press, Cambridge, MA, 1021-1042.

29. Andersen RA, Snyder LH, Bradley DC \& Xing J (1997). Multimodal representation of space in the posterior parietal cortex and its use in planning movements. Annual Review of Neuroscience, 20: 303330.

30. Kaas J H (2000). The reorganization of somatosensory and motor cortex after peripheral nerve or spinal cord injury. Progress in Brain Research, 128: 173-179.

31. Fiorani J unior M, Rosa MG, Gattass R \& Rocha-Miranda CE (1992). Dynamic surrounds of receptive fields in primate striate cortex: a physiological basis for perceptual completion? Proceedings of the National Academy of Sciences, USA, 89: 8547-8551.

32. von der Heydt R \& Peterhans E (1989). Mechanisms of contour perception in monkey visual cortex. I. Lines of pattern discontinuity. J ournal of Neuroscience, 9: 1731-1748.

33. De Weerd P, Gattass R, Desimone R \& Ungerleider LG (1995). Responses of cells in monkey visual cortex during perceptual filling-in of an artificial scotoma. Nature, 377: 731-734.

34. Rossi AF, Rittenhouse CD \& Paradiso MA (1996). The representation of brightness in primary visual cortex. Science, 273: 1104-1107.

35. Lamme VAF \& Spekreijse H (2000). Contextual modulation in primary visual cortex and scene perception. In: Gazzaniga MS (Editor), The New Cognitive Neurosciences. 2nd edn. MIT Press, Cambridge, MA, 279-290.

36. Huk AC \& Heeger DJ (2000). Task-related modulation of visual cortex. J ournal of Neurophysiology, 83: 3525-3536.

37. Watanabe $T$, Harner AM, Miyauchi $S$, Sasaki Y, Nielsen M, Palomo D \& Mukai I (1998). Task-dependent influences of attention on the activation of human primary visual cortex. Proceedings of the National Academy of Sciences, USA, 95: 11489-11492.

38. Ramachandran VS \& Rogers-Ramachandran D (1996). Synesthesia in phantom limbs induced with mirrors. Proceedings of the Royal Society of London. B, Biological Sciences, 263: 377-386. 
39. Montoya P, Ritter K, Huse E, Larbig W, Braun C, Töpfner S, Lutzemberger V, Grood W, Flor H \& Birbaumer N (1998). The cortical somatotopic map and phantom phenomena in subjects with congenital limb atrophy and traumatic amputees with phantom limb pain. European J ournal of Neuroscience, 10: 1095-1102.

40. Brugger P, Kollias SS, Müri RM, Crelier G, Hepp-Reymond MC \& Regard M (2000). Beyond remembering: Phantom sensations of congenitally absent limbs. Proceedings of the National Academy of Sciences, USA, 97: 6167-6172.

41. Ramachandran VS \& Hirstein W (1998). The perception of phantom limbs. The D.O. Hebb Lecture. Brain, 121: 16031630.

42. Mon-Williams $M$, Wann J P, J enkinson $M$ \& Rustioni A (1997). Synesthesia in the normal limb. Philosophical Transactions of the Royal Society of London, Series B. Biological Sciences, 264: 1007-1010.

43. Zangaladze A, Epstein CM, Grafton ST \& Sathian K (1999). Involvement of visual cortex in tactile discrimination of orientation. Nature, 401: 587-590.

44. Sathian K, Zangaladze A, Hoffman J M \& Grafton ST (1997). Feeling with the mind's eye. NeuroReport, 8: 3877-3881.

45. Ramachandran VS, Levi L, Stone L, Rogers-Ramachandran D, McKinney R, Stalcup M, Arcilla G, Zweifler R, Schatz A \& Flippin A (1996). Illusions of body image: What they reveal about human nature. In: Llinás R \& Churchland PS (Editors), The Mind-Brain Continuum. Sensory Processes. MIT Press, Cambridge, MA, 29-60.

46. Fregnac $Y$, Shulz $D$, Thorpe $S \&$ Bienenstock $E$ (1988). A cellular analogue of visual cortical plasticity. Nature, 333: 367370.

47. Fregnac $Y$, Shulz $D$, Thorpe $S \&$ Bienenstock $E$ (1992). Cellular analogues of visual cortical epigenesis. I. Plasticity orientation selectivity. J ournal of Neuroscience, 12: 1280-1300.

48. Recanzone $\mathrm{GH}$, Merzenich $\mathrm{MM}$, J enkins WM, Grajski KA \& Dinse HR (1992). Topographic reorganization of the hand representation in cortical area $3 \mathrm{~b}$ of owl monkeys trained in a frequency-discrimination task. J ournal of Neurophysiology, 67: 1031-1056.
49. Crist RE, Kapadia MK, Westheirmer G \& Gilbert CD (1997). Perceptual learning of spatial localization: specificity for orientation, position, and context. J oumal of Neurophysiology, 78: 2889-2894.

50. Crist RE, Li W \& Gilbert CD (2001). Leaming to see: experience and attention in primary visual cortex. Nature Neuroscience, 4: 519-525.

51. Kami A \& Sagi D (1993). The time course of learning a visual skill. Nature, 365: 250252.

52. Luiten PGM, Gaykema RPA, Traber J \& Spencer DG (1987). Cortical projection pattems of magnocellularis basal nucleus subdivisions as revealed by anterograde transported Phaseolus vulgaris leucoagglutinin. Brain Research, 413: 229-250.

53. McGaugh J \& Sarter M (1998). Sustained attention performance in rats with intracortical infusions of 192 lgG-saporin-induced cortical cholinergic deafferentation: effects of physostigmine and FG 7142. Behavioral Neuroscience, 112: 15191525.

54. Moro V, Badaut J, Springhetti V, Edvinsson L, Seylaz J \& Lasbennes F (1995). Regional study of the co-localization of neuronal nitric oxide synthase with muscarinic receptors in the rat cerebral cortex. Neuroscience, 69: 797-805.

55. Smiley JF, Levey Al \& Mesulam M-M (1998). Infracortical interstitial cells concurrently expressing M2-muscarinic receptors, acetylcholinesterase and nicotinamide adenine dinucleotide phosphatediaphorase in the human and monkey cerebral cortex. Neuroscience, 84: 755-769.

56. Franca J G, Volchan E, J ain N, Catania KC, Oliveira RLS, Hess FF, J ablonka M, RochaMiranda CE \& Kaas JH (2000). Distribution of NADPH-diaphorase cells in visual and somatosensory cortex in four mammalian species. Brain Research, 864: 163175.

57. Bredt DS \& Snyder SH (1992). Nitric oxide, a novel neuronal messenger. Neuron, 8: 3-11.

58. Hölscher C (1997). Nitric oxide, the enigmatic neuronal messenger: its role in synaptic plasticity. Trends in Neurosciences, 20: 298-303.

59. Schuman EM \& Madison DV (1994). Nitric oxide and synaptic function. Annual Review of Neuroscience, 17: 153-183.
60. Diamond DM \& Weinberger NM (1989). Role of context in the expression of learning-induced plasticity of single neurons in auditory cortex. Behavioral Neuroscience, 103: 471-494.

61. Bakin J S \& Weinberger NM (1990). Classical conditioning induces CS-specific receptive field plasticity in the auditory cortex of the guinea pig. Brain Research, 536: 271-286.

62. Edeline JM \& Weinberger NM (1993). Receptive field plasticity in the auditory cortex during frequency discrimination training: selective retuning independent of task difficulty. Behavioral Neuroscience, 107: 82-103.

63. Lennartz RC \& Weinberger NM (1992). Frequency-specific receptive field plasticity in the medial geniculate body induced by Pavlovian fear conditioning is expressed in the anesthetized brain. Behavioral Neuroscience, 106: 484-497.

64. Weinberger NM, J avid R \& Lepan B (1993). Long-term retention of learninginduced receptive-field plasticity in the auditory cortex. Proceedings of the National Academy of Sciences, USA, 90: 23942398.

65. Weinberger NM (1998). Physiological memory in primary auditory cortex: characteristics and mechanisms. Neurobiology of Leaming and Memory, 70: 226251.

66. LeDoux J (1996). The Emotional Brain. Simon \& Schuster, New York.

67. Vazdarjanova A \& McGaugh JL (1999). Basolateral amygdala is involved in modulating consolidation of memory for classical fear conditioning. J ournal of Neuroscience, 19: 6615-6622.

68. Dykes RW (1997). Mechanisms controlling neuronal plasticity in somatosensory cortex. Canadian J ournal of Physiology and Pharmacology, 75: 535-545.

69. Gold PE, Weinberger NM \& Stemberg DB (1985). Epinephrine-induced learning under anesthesia: retention performance at several training-testing intervals. Behavioral Neuroscience, 99: 1019-1022.

70. Weinberger NM, Gold PE \& Stemberg DB (1984). Epinephrine enables Pavlovian fear conditioning under anesthesia. Science, 223: 605-607. 